\title{
Elevated Serum Aminotransferases Secondary to Rippling Muscle Disease
}

\author{
Kumanan Nalankillia ${ }^{\mathrm{a}}$ John Lubel $^{\mathrm{b}}$ \\ ${ }^{a}$ Department of Gastroenterology and Hepatology, Eastern Health, Melbourne, Vic; \\ ${ }^{b}$ Department of Gastroenterology and Hepatology, Eastern Health, Melbourne, Vic., and \\ Eastern Health Clinical School, Monash University, Melbourne, Vic., Australia
}

\section{Key Words}

Aminotransferase $\cdot$ Caveolin-3 $\cdot$ Clinical decision making $\cdot$ Rippling muscle disease $\cdot$ Liver tests

\begin{abstract}
A 43-year-old man was referred by his general practitioner to the hepatology clinic with deranged serum aminotransferases, discovered as part of routine blood tests. The objective was to identify the cause of elevated serum aminotransferases in this patient in a systematic manner. Thorough history and physical examination revealed a background history of rippling muscle disease secondary to caveolin-3 protein deficiency, with typical clinical signs. There was a positive family history of musculoskeletal disease in the patient's father and brother. Previous diagnostic tests performed to investigate the patient's musculoskeletal symptoms, including muscle biopsies, were revisited. Subsequent systematic investigations such as blood tests, liver ultrasound scan and Fibroscan ${ }^{\circledR}$ were performed to exclude potential causes of the deranged serum aminotransferases. Liver biopsy was not performed. A consistent pattern of chronic low-grade elevations of serum aminotransferases, less than three times the upper limit of the normal range, was found. This was associated with a consistently elevated serum creatine kinase and normal renal function tests. Previous muscle biopsies had revealed chronic degenerative and regenerative changes suggestive of a focal necrotizing myopathy. Liver ultrasound scan and Fibroscan ${ }^{\circledR}$ were normal. With exclusion of other liver diseases and identification of profoundly elevated serum creatine kinase concentration, the deranged aminotransferases were attributed to rippling muscle disease.
\end{abstract}


Nalankilli et al.: Elevated Serum Aminotransferases Secondary to Rippling Muscle Disease

\section{Introduction}

Deranged liver tests are a common reason for patients to be referred to hepatologists. A systematic approach when further investigating such patients is important to ensure that primary liver disease is excluded, rarer causes are not overlooked, and unnecessary invasive investigations are avoided. This case illustrates that elevated serum aminotransferases may be derived from tissues other than hepatocytes, such as skeletal muscle cells by using the example of a rare muscle disorder, rippling muscle disease.

\section{Case Report}

\section{Presentation}

A 43-year-old male fitness trainer presented to the hepatology clinic after being referred by his general practitioner with elevated serum aspartate aminotransferase (AST) and alanine aminotransferase (ALT) levels (2-3 times the upper limit of normal) over a period of 7 months. Bilirubin, gamma-glutamyl transpeptidase (GGT) and alkaline phosphatase (ALP) were within normal range. On further questioning, the patient gave a history of rippling muscle disease diagnosed when he was 9 years old. Subsequently, he had been lost to further follow-up. There was no other relevant medical history. Specifically he had no history of alcohol abuse or dependency, no history of illicit drug use, no known history of hepatitis and he was not on any regular medications. A detailed family history and research into previous investigations performed on the patient and his family revealed that his father and elder brother had been found to have elevated serum creatine kinase (CK) concentrations. Genetic linkage analysis had pointed to caveolin-3 mutations in all of them.

On physical examination, there were no stigmata of chronic liver disease, and gastrointestinal, cardiovascular and respiratory examinations were unremarkable. Positive neuromuscular findings consistent with rippling muscle disease included a long-standing history of activity-related fatigue of upper and lower limb muscle groups. He had obvious symmetrical wasting of pectoralis major muscles bilaterally (fig. 1). Characteristic muscle rippling was demonstrated after minimal activity of proximal muscles in the upper limbs and lower limbs bilaterally (online suppl. video clips 1 and 2; for all online suppl. material, see www.karger.com/doi/10.1159/000351824). He demonstrated fatigue with repetitive contractions of major skeletal muscle groups (online suppl. video clip 3).

\section{Investigations}

Full blood count examination and serum urea and electrolytes examination were normal. Full liver screen including a liver ultrasound, viral hepatitis, cytomegalovirus screen and autoimmune screen were also normal. There was no evidence of haemochromatosis or Wilson's disease with normal iron studies, serum copper and caeruloplasmin. Thyroid function tests were normal. A Fibroscan ${ }^{\circledR}$ of the liver demonstrated median stiffness of $5.9 \mathrm{kPa}$ consistent with minimal or absent fibrosis.

Previous diagnostic studies including a gastrocnemius muscle biopsy when the patient was 9 years of age showed predominance of type 1 fibres, with isolated fibres exhibiting atrophy. There were also degenerative and regenerative changes, suggestive of mild focal necrotising myopathy.

Blood tests over a 10-month period are shown in table 1. A consistent elevation of serum aminotransferases and CK is demonstrated. 


\begin{tabular}{l|l}
\hline \multicolumn{2}{l}{ Case Rep Gastroenterol 2013;7:234-239 } \\
\hline DOI: $10.1159 / 000351824$ & $\begin{array}{l}\text { C 2013 S. Karger AG, Basel } \\
\text { www.karger.com/crg }\end{array}$ \\
\hline
\end{tabular}

Nalankilli et al.: Elevated Serum Aminotransferases Secondary to Rippling Muscle Disease

\section{Differential Diagnosis}

ALT and AST catalyse the transfer of the alpha-amino groups of alanine and aspartate, respectively, to the alpha-keto group of ketoglutarate. This results in the formation of pyruvate and oxaloacetate [1].

Various studies have analysed the clinical significance of asymptomatic deranged serum aminotransferases $[2,3]$. In a British study of patients where the diagnosis was not evident with history, physical examination and non-invasive tests including ultrasound scans, the most common diagnoses were found to be non-alcoholic fatty liver disease and alcoholic liver disease [2]. In another prospective study where patients went on to have a liver biopsy, steatosis and steatohepatitis were the most common diagnoses [3]. Results have tended to vary depending on the prevalence of liver disease in the population groups studied and the extent to which an underlying cause for these abnormalities was explored. For example, in a study of American blood donors, a diagnosis could not be established in the majority of patients with incidental elevated ALT [4]. This was partly attributed to the limited investigations and diagnostic algorithm used in the study.

In the setting of elevated serum aminotransferases (less than five times the normal range), the following investigative steps can be derived from the American Gastroenterological Association recommendations [5, 6]: (1) Thorough history and physical examination. (2) Identify medications and supplements that can cause derangement and discontinue them. Assess for alcohol use, viral hepatitis B and C, haemochromatosis and fatty liver disease. (3) Identify non-hepatic causes (primary muscle disease, hypo- and hyperthyroidism, coeliac disease, adrenal insufficiency and anorexia nervosa). (4) Identify rarer liver conditions such as autoimmune hepatitis, Wilson's disease, alpha-1-antitrypsin deficiency and adult bile ductopenia. (5) Proceed to a liver biopsy (patients with greater than two-fold elevation in aminotransferases) or observe over a period of time (patients with less than two-fold elevation in aminotransferases) before liver biopsy.

However, the decision to pursue specific investigations should be based on the pattern of abnormality of liver tests, and be directed by features obtained from history, physical examination and pre-test probability. In this case, elevated serum aminotransferases with a normal serum GGT and ALP indicated a hepatocellular pattern of injury as opposed to cholestasis. Non-invasive investigations performed on the patient did not reveal a hepatic cause, but a detailed history raised the distinct possibility of a non-hepatic cause. The patient gave a history of long-standing muscle fatigue since childhood and consistently exhibited typical signs of rippling muscle disease, such as the rippling phenomenon. Serum aminotransferases were elevated in conjunction with serum CK, which is a known marker of muscle injury.

Serum aminotransferase levels reflect the rate at which they enter and are cleared from the circulation. They are catabolised by proteolysis in the reticulo-endothelial system and are not excreted in significant amounts in urine and bile [7]. Hence, derangement is usually related to damage to tissues rich in the aminotransferases, or to changes in cell membrane permeability that permit leakage into the circulation [7].

Although hepatocytes contain the highest concentration of ALT and AST, it is well established that they are also present in other tissues such as cardiac and skeletal muscle [1]. Because their isoenzymes are not tissue-specific, isoenzyme measurements of ALT and AST do not aid in identifying the tissue of origin accurately [7]. In this clinical setting, chronic degeneration and regeneration of muscle fibres that were demonstrated in the patient's muscle biopsy causing leakage of these enzymes into the circulation explain the chronic low-grade elevation of aminotransferases. 
Nalankilli et al.: Elevated Serum Aminotransferases Secondary to Rippling Muscle Disease

\section{Discussion}

Caveolin-3 is a protein that forms flask-shaped invaginations called caveolae on the cytoplasmic surface of the sarcolemma. It is composed of 151 amino acids and is synthesised in the endoplasmic reticulum of muscle cells. Caveolae stabilise the sarcolemma and facilitate signal transduction and vesicular transport across it [8]. Caveolin-3 is encoded by the CAV-3 gene, which is mapped on chromosome 3p25. Mutations in this gene result in underexpression of the caveolin-3 protein $[8,9]$.

Four known skeletal muscle phenotypes have been attributed to the various caveolin-3 mutations identified to date [8]. They are: (1) Limb-girdle muscular dystrophy type 1C characterised by weakness and wasting restricted to the proximal limb-girdle musculature. (2) Isolated hyperCKaemia - no clinical manifestations. (3) Rippling muscle disease - characterised by signs such as percussion-induced rapid contractions and stretch-induced muscle mounding or rippling. Other clinical features may include muscle fatigue, muscle stiffness, tip-toe walking and myalgia [10]. (4) Distal myopathy - characterised by weakness and wasting restricted to the distal limb musculature.

Muscle biopsies may show characteristic features such as muscle fibre degeneration and regeneration, a predominance of type 1 fibres and fibre size variability. Elevated CK levels have been noted in all four phenotypes [8].

Patients may exhibit significant overlap between these phenotypes, and some suggest that they be seen as a clinical continuum $[8,11]$. In our patient, the characteristic rippling phenomenon demonstrated clinically, genetic linkage analyses and suggestive findings in the muscle biopsy pointed to the diagnosis of rippling muscle disease. Immunohistochemistry was not performed as he had been lost to follow-up in later years. However, it was noted that the patient's father and brother had elevated CK levels, but were relatively asymptomatic. This illustrates the phenotypic variability amongst individuals with the same mutation, suggesting incomplete penetrance.

To date, there are no case reports on deranged liver tests as a result of caveolin-3 deficiency-related muscle disease, though there are reports of elevated aminotransferases in other more common muscle diseases. For example, Tay et al. [12], in a case series analysis study done in Singapore, showed that aminotransferases were elevated in all patients in their cohort with biopsy-proven Duchenne or Becker's muscular dystrophy and that their levels were inversely proportional to their extent of mobility and age. This case adds to the existing medical literature in highlighting a different muscle pathophysiology driving similar laboratory results. Along with muscle degeneration, changes in the sarcolemmal architecture secondary to caveolin-3 underexpression could also contribute to the chronic leakage of aminotransferases into the circulation. This could be a subject for future research.

Caveolin-3 deficiency has been implicated in one case series of familial hypertrophic cardiomyopathy [13] and some instances of arrhythmogenic long QT syndrome [8]. The current consensus on caveolin-3-deficient muscle diseases is that patients need symptomatic care with the aim to preserve muscle function. Regular monitoring of respiratory and cardiac function is also recommended on a case by case basis [8].

It was explained to the patient that the cause of his deranged liver tests was in fact chronic muscle disease. Given that it seemed to demonstrate an autosomal dominant pattern of inheritance, he was referred to a clinical genetics specialist for genetic counselling and it was suggested that his offspring be tested for caveolin-3 deficiency. The outcome of the consultation and our recommendations were communicated to the patient's general practitioner. 
Nalankilli et al.: Elevated Serum Aminotransferases Secondary to Rippling Muscle Disease

\section{Acknowledgements}

Informed consent for publication was obtained from the patient who is the subject of this case report. We wish to thank him for his contribution.

\section{References}

1 Rosalki SB, McIntyre N: Biochemical investigations in the management of liver disease; in Bircher J, Benhamou JP, McIntyre N, et al (eds): Oxford Textbook of Clinical Hepatology, ed 2, vol 1. New York, Oxford University Press, 1999, pp 506-507.

-2 Armstrong MJ, Houlihan DD, Bentham L, et al: Presence and severity of non-alcoholic fatty liver disease in a large prospective primary care cohort. J Hepatol 2012;56:234-240.

-3 Daniel S, Ben-Menachem T, Vasudevan G, et al: Prospective evaluation of unexplained chronic liver transaminase abnormalities in asymptomatic and symptomatic patients. Am J Gastroenterol 1999;94: 3010-3014.

-4 Kundrotas LW, Clement DJ: Serum alanine aminotransferase (ALT) elevation in asymptomatic US Air Force basic trainee blood donors. Dig Dis Sci 1993;38:2145-2150.

5 Green RM, Flamm S: AGA technical review on the evaluation of liver chemistry tests. Gastroenterology 2002;123:1367-1384.

6 Friedman LS: Approach to the patient with abnormal liver function tests, 2012. http://www.uptodate.com/ contents/approach-to-the-patient-with-abnormal-liver-function-tests (accessed September 2012).

7 Friedman LS: Liver function tests that detect injury to hepatocytes, 2010. http://www.uptodate.com/ contents/liver-function-tests-that-detect-injury-to-hepatocytes (accessed September 2012).

8 Gazzerro E, Sotgia F, Bruno C, et al: Caveolinopathies: from the biology of caveolin-3 to human diseases. Eur J Hum Genet 2010;18:137-145.

-9 Traverso M, Gazzerro E, Assereto S, et al: Caveolin-3 T78M and T78K missense mutations lead to different phenotypes in vivo and in vitro. Lab Invest 2008;88:275-283.

10 Torbergsen T: Rippling muscle disease: a review. Muscle Nerve Suppl 2002;11:S103-S107.

-11 Kubisch C, Schoser B, Von During M, et al: Homozygous mutations in caveolin-3 cause a severe form of rippling muscle disease. Ann Neurol 2003;53:512-520.

12 Tay SK, Ong HT, Low PS: Transaminitis in Duchenne's muscular dystrophy. Ann Acad Med Singapore 2000;29:719-722.

13 Hayashi T, Arimura T, Ueda K, et al: Identification and functional analysis of a caveolin-3 mutation associated with familial hypertrophic cardiomyopathy. Biochem Biophys Res Commun 2004;313:178-184.

Table 1. Liver tests and serum CK between August 2011 and June 2012

\begin{tabular}{|c|c|c|c|c|c|}
\hline Parameter (reference range) & $30 / 08 / 2011$ & $03 / 11 / 2011$ & $20 / 12 / 2011$ & $16 / 03 / 2012$ & $28 / 06 / 2012$ \\
\hline Bilirubin $(4-20 \mu \mathrm{mol} / \mathrm{l})$ & 5 & 4 & 4 & 5 & 5 \\
\hline ALT (5-40 U/l) & 91 & 126 & 103 & 87 & 68 \\
\hline AST (10-40 U/l) & 70 & 113 & 104 & 73 & 56 \\
\hline GGT (5-50 U/l) & 17 & 16 & 19 & 22 & 18 \\
\hline ALP (35-110 U/l) & 46 & 51 & 52 & 54 & 54 \\
\hline Albumin $(39-50 \mathrm{~g} / \mathrm{l})$ & 45 & 45 & 44 & 45 & 40 \\
\hline Total protein $(66-83 \mathrm{~g} / \mathrm{l})$ & 76 & 73 & 75 & 77 & 71 \\
\hline CK (60-220 IU/l) & not done & not done & not done & 3,564 & 3,738 \\
\hline Urea $(2.5-8.5 \mathrm{mmol} / \mathrm{l})$ & 5.5 & 6.0 & 6.3 & 5.3 & 6.1 \\
\hline Creatinine $(50-100 \mu \mathrm{mol} / \mathrm{l})$ & 62 & 65 & 67 & 60 & 63 \\
\hline
\end{tabular}


Nalankilli et al.: Elevated Serum Aminotransferases Secondary to Rippling Muscle Disease

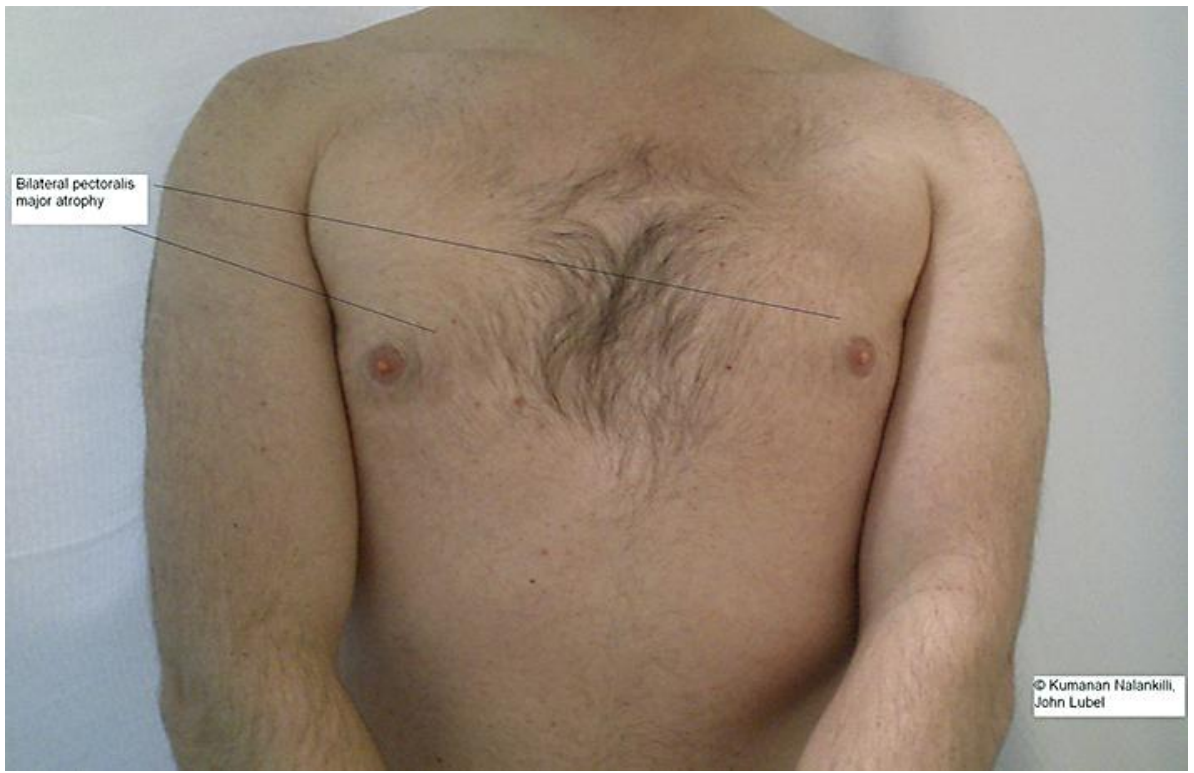

Fig. 1. Bilateral pectoralis major atrophy demonstrated in an individual with rippling muscle disease. 